\section{Phenotypic and genetic complexity of psychosis}

\author{
Invited commentary on ... Schizophrenia: a common disease \\ caused by multiple rare alleles ${ }^{\dagger}$
}

NICK CR ADDOCK, MICHAEL C. O'DONOVAN and MICHAEL J. OWEN

\begin{abstract}
Summary Psychosis, like other major psychiatric disorders, is both genetically and clinically complex. Increasingly powerful molecular genetic studies have the potential to identify DNA variation that influences susceptibility to genetically complex disorders. There is a need to use a range of genetic approaches appropriate to identifying a spectrum of risk variants from the common through to the rare. Some variants might have large effects at the level of the individual but most are likely to have modest or small effects at both population and individual level.

Extensive clinical heterogeneity is likely to have a significant impact on the power of even the largest studies and, more importantly, will lead to extensive variability between studies and hamper attempts at replication. If we are to realise the potential of molecular genetics, we need to overcome the major limitations imposed by current psychiatric diagnostic classifications and identify clinical phenotypes that reflect the presence of underlying entities with biological validity.
\end{abstract}

\section{Declaration of interest None.}

In this month's Journal McClellan et al contrast two models of the genetic architecture of schizophrenia. Here we provide a context for that paper by considering more widely the genetic and phenotypic complexity of psychosis and how this has an impact on genetic research.

\section{COMPLEX GENETIC DISEASES}

The term 'complex genetic diseases' refers to common familial illnesses that do not

\footnotetext{
†See pp. 194-199, this issue.
}

show a simple Mendelian pattern of inheritance (Lander \& Schork, 1994). Examples include coronary heart disease, hypertension, rheumatoid arthritis, type I and type II diabetes mellitus, asthma, many cancers and most psychiatric disorders. In terms of their genetic properties and complexity, psychiatric disorders, including schizophrenia and bipolar disorder, are very similar to the non-psychiatric common familial disorders. In fact, perhaps surprisingly, genetic susceptibility to risk is substantially higher for the major psychiatric illnesses than for most of the nonpsychiatric diseases (Plomin et al, 1994). What makes the study of psychiatric genetics substantially more difficult than investigation of the complex non-psychiatric diseases is the lack of biologically valid measures for phenotype definition.

\section{RARE VARIANTS OF LARGE EFFECT AND COMMON VARIANTS OF SMALL EFFECT}

\section{Theory}

McClellan et al consider two distinct genetic models that can explain the transmission of common familial disorders that show non-Mendelian inheritance. They conclude that most cases of schizophrenia are likely to be explained by genetic variants of large effect that, although individually rare, in their totality account for the majority of cases in the population. According to this view, only one rare variant of large effect is involved in each family, but different variants, which may be in the same or in other genes, operate in other families. This is sometimes called the 'common disease-rare variant' model. It can be contrasted with the 'common disease-common variant' model which forms the rationale for the large-scale genetic association studies that are ongoing in many centres around the world. In the latter model, a common disease, such as schizophrenia, results from the coaction of multiple (ranging in principle from a few to many thousand) common variants ('polymorphisms'), each of which has a small effect on illness susceptibility. When an individual inherits several, or many, susceptibility variants together, they have a sizable influence on disease risk. This is essentially the traditional 'multifactorial' model that assumes the action of multiple genes and environmental risk factors (Falconer \& MacKay, 1995).

\section{Schizophrenia}

McClellan et al argue strongly against the common disease-common variant model but argue in favour of rare variants of large effect. Since we still do not know the true genetic architecture of schizophrenia, challenges to widely held assumptions and discussions of the possible impact on gene discovery are welcome. However, a considerable body of genetic epidemiological and molecular data relating to schizophrenia as well as population genetic findings do allow some inferences to be drawn and constrain the nature of plausible models. We agree with McClellan et al that rare mutations are likely to be important in some cases of schizophrenia; there are indeed examples in which schizophrenia is related to chromosomal abnormalities. However, it is our contention that key genetic epidemiological and molecular genetic observations are inconsistent with the hypothesis that rare variants of large effect can explain the majority of cases of schizophrenia. Further, the dismissal by McClellan and colleagues of the importance of variants of modest or small effect is not well founded. Important pieces of evidence that contradict their assertions are given below.

\section{Families with clear Mendelian} inheritance patterns are rare

Under the model of rare variants of major effect, even allowing for a high proportion of new mutations, it would be expected that there would be many families with clear-cut single gene inheritance. However, as experienced clinical psychiatrists will know, such families are rare.

\section{Single genes of major effect} have not been found

Over the past 20 years hundreds of diseases with Mendelian inheritance have been subjected to genetic analysis ('positional cloning') which has allowed detection of mutations of major effect using only a few 
families or just one large pedigree (Collins, 1992; Botstein \& Risch, 2003), some of which are cited by McClellan et al. Although rare, extended pedigrees multiply affected by psychosis do exist and have been studied genetically. If single genes of major effect explained illness in such pedigrees, the genetic methods used should have identified them, or at least unambiguously defined a chromosomal location, as has been done successfully for the many disorders with Mendelian inheritance. However, when extended pedigrees with multiple cases of illness that are consistent with simple Mendelian inheritance patterns have been subjected to intensive molecular genetic study, not only have mutations of major effect not been identified, the evidence for linkage is much weaker than one would expect if the families were segregating a single cause of the disorder. Rather, findings to date are consistent with multiple variants of modest effect (see Chumakov et al, 2002; Stefansson et al, 2002; Straub et al, 2002).

\section{Mathematical modelling of familial risk is inconsistent with single genes of large effect}

McClellan et al cite Risch's studies modelling the way risk of illness changes as a function of genetic relatedness to a sufferer (Risch, 1990). For both schizophrenia and bipolar disorder there is a very rapid, nonlinear decrease of risk when moving from a genetically identical individual (i.e. monozygotic co-twin where the risk is $50-60 \%$ ), to an individual who shares half the genes (e.g. sibling, parent, dizygotic co-twin where risk is around $10 \%$ ). Contrary to the assertion by McClellan et al, mathematical modelling demonstrates that this pattern cannot be explained by a collection of genes of large effect that act on their own, even if a sizable proportion are de novo mutations. For illnesses where one mutation is a sufficient cause of illness in each family (whether or not there is a different mutation or gene involved in different families) there is a more gradual (linear) decrease of risk (McGue \& Gottesman, 1989). In contrast, the rapid, non-linear decrease of risk is compatible with multiple interacting risk factors, albeit of unknown frequency, that individually have modest effects (Risch, 1990; Craddock et al, 1995).

Molecular genetic findings are consistent with multiple risk alleles of modest effect

Several genes have been implicated repeatedly as conferring risk for schizophrenia or bipolar disorder. These include dysbindin (DTNBP1) (Straub et al, 2002; Williams et al, 2005), neuregulin 1 (NRG1; Stefansson et al, 2002; Tosato et al, 2005; Munafo et $a l, 2006)$ and D-amino acid oxidase activator (DAOA, G72/G30; Chumakov et al, 2002; Detera-Wadleigh \& McMahon, 2006). The patterns of effect sizes and allele frequencies are consistent with the common diseasecommon variant model and with the positive findings that have been emerging in studies of non-psychiatric complex genetic diseases (Todd, 2006). Estimated effect sizes are all modest, with estimated relative risks (or odds ratios) typically below 2.0. In contrast, no rare alleles of large effect have yet been unequivocally identified, although a few rare chromosomal aberrations have been shown to dramatically increase risk (Craddock et al, 2005).

\section{GENETIC COMPLEXITY OF SCHIZOPHRENIA}

As is often the case with dichotomous decisions, choosing between either rare variants of large effect or common variants of small effect is almost certainly oversimplistic. Instead, it is probably more reasonable to assume that the spectrum of mutations for common disease is similar to that of all variants in the human genome. This leads to the expectation of a spectrum of risk variants of varying effect sizes, including both common and rare alleles (Wang et al, 2005). Note that the spectrum of likely risk alleles also includes rare variants of small or modest effect size, the existence of which might well prove to be a far greater obstacle to gene discovery than rare alleles of large effect size.

It is important to acknowledge that, in addition to the models already discussed, several molecular genetic mechanisms are known that result in complex, nonMendleian patterns of inheritance for a disorder or trait. Examples include: dynamic mutations (e.g. the expanding trinucleotide repeats that underlie fragile $\mathrm{X}$ disorder); genomic imprinting (e.g. Prader-Willi syndrome); and mitochondrial inheritance (e.g. some optic atrophies) as well as other mechanisms involving deletion, insertion or variable repetition of stretches of DNA. Such mechanisms might contribute to the genetic complexity of psychiatric illness and need to be considered in the search for genetic factors that influence susceptibility to schizophrenia (see Margolis et al, 1999; Malaspina, 2001; Singh et al, 2002; Ben-Shachar \& Laifenfeld, 2004).

\section{PHENOTYPIC COMPLEXITY IN PSYCHIATRIC ILLNESS}

For most complex genetic diseases, although pathophysiology is incompletely understood, there are biological measures that can be used to define the phenotype (e.g. blood glucose in diabetes, blood pressure in hypertension or biopsy and histology for cancers). These measures are typically reliable and, importantly, have biological validity. The fundamental importance of phenotype definition and measurement for the success of gene identification in human genetics has long been appreciated (Lander \& Schork, 1994). It is surprising, therefore, that the vast majority of psychiatric genetic studies continue to rely on DSM-IV (or ICD-10) diagnostic categories as if they were proven, valid disease entities. Many researchers have assumed that the effects of heterogeneity would be overcome as the technical advances of molecular genetics allowed increasingly large and powerful studies. However, this might not occur if, as well might be the case, researchers tend to adopt less restrictive, or more 'pragmatic', inclusion criteria to facilitate the assembly of larger samples. Genetic research might benefit more from the use of smaller, more homogeneous than larger, more heterogeneous samples (Craddock et al, 2006). This principle is clearly demonstrated by the example of the D-amino acid oxidase activator gene $(D A O A)$ which has been implicated in some, but not all, studies of both schizophrenia and bipolar disorder (Chumakov et al, 2002; Hattori et al, 2003; Detera-Wadeleigh \& McMahon, 2006). In a large study we found evidence that the gene confers risk for episodes of pathological mood disturbance irrespective of diagnostic category (Williams et al, 2006). We found significant association in 706 individuals meeting DSM-IV criteria for bipolar disorder. Among those meeting criteria for schizophrenia there was significant association in 112 who had also experienced major mood episodes but not in 597 without major mood episodes. Further, no association was detectable if the schizophrenia sample was treated as a single homogeneous entity (as is the case in most studies). This suggests that results of studies of DAOA in categorically defined 'schizophrenia' samples are dependent upon the proportion of people in the sample that have experienced mood disorder - information that is not usually provided or considered by researchers. 
Table I Factors affecting issues of genetic and phenotypic complexity in psychiatric genetic studies

\begin{tabular}{|c|c|c|}
\hline \multirow[b]{2}{*}{ Issue } & \multicolumn{2}{|c|}{ Factors affecting } \\
\hline & Clinical phenotype & Genotype \\
\hline \multirow[t]{3}{*}{ Population studied } & Environment & Genetic differences \\
\hline & Sociocultural factors & Population stratification/'structure' \\
\hline & Service provision & \\
\hline \multirow[t]{5}{*}{ Participant ascertainment } & Severity & Genetic loading \\
\hline & Symptom pattern & Selection for simple inheritance \\
\hline & Course of illness & \\
\hline & Impairment & \\
\hline & Treatment response & \\
\hline Underlying model & $\begin{array}{l}\text { Unknown phenotype (disease) model } \\
\text { requires analytical strategies that do } \\
\text { not rely on knowing model precisely }\end{array}$ & $\begin{array}{l}\text { Unknown genetic model requires } \\
\text { analytical strategies that do not rely } \\
\text { on knowing model precisely }\end{array}$ \\
\hline \multirow[t]{3}{*}{ Measurement } & Consistency of assessment methods & Consistency of genotyping methods \\
\hline & Variable use of standard terminology & Genotyping error \\
\hline & Phenotype measurement error & \\
\hline
\end{tabular}

A further striking example is provided by the gene Disrupted in Schizophrenia 1 (DISC1). This was identified by studies of an extended Scottish pedigree in which a spectrum of psychiatric illness, including mood and psychotic diagnoses, co-segregated with a chromosomal translocation (St Clair et al, 1990; Millar et al, 2000; Muir et al, 2006). Although the name given to the gene by the research team explicitly refers to schizophrenia, major disorder is actually more strongly linked to the translocation (Blackwood et al, 2001). We have provided independent evidence from a linkage study of families with schizoaffective disorder that variation at the DISC1 locus influences susceptibility to psychopathology involving disturbances in both mood and psychotic domains (Hamshere et al, 2005).

All the molecular evidence suggests that genetic susceptibility does not respect current operational diagnostic boundaries (Craddock \& Owen, 2005). This will not surprise psychiatrists. 'Schizophrenia' is so broad that it is possible for one sample to be composed of individuals with chronic disability involving cognitive impairment, marked negative features and minimal affective or positive psychotic symptoms whereas another sample could include individuals who are able to function relatively well, with an episodic course and marked affective and positive psychotic symptoms. Self-evidently, unless clinical variation is the consequence of chance or of environmental risk factors, illness in each of the above two samples will reflect the operation of at least some susceptibility alleles not held in common at the same frequency in each group. Since the key to unambiguously identifying a risk factor is replication across different samples, we must move beyond diagnostic categories for describing and analysing samples and routinely consider more detailed measures of lifetime psychopathology. There are substantial theoretical benefits of using endophenotypes (intermediate phenotypes) such as neuroimaging or tests of cognitive function to define more homogeneous groups or to access more directly abnormalities that mediate the effects of genes on psychopathology (Jablensky, 2006; Braff et al, 2007). However, these approaches are not without difficulties (Owen et al, 2005) and most samples collected to date for genetic studies have clinical data rather than these extended measures. It is therefore relatively simple and inexpensive to make more effective use of the clinical data that can be used to characterise individuals.

\section{ISSUES THAT AFFECT PHENOTYPIC COMPLEXITY}

Much attention has been devoted to genetic issues that can affect comparisons between studies. These include genetic differences between different geographical or ethnic populations (so-called population stratification or structure; see Cardon \& Palmer, 2003), methods of ascertainment (see McCarthy et al, 1998), approaches to dealing with unknown genetic models (see Risch, 2000), and genotyping error (see Moskvina et al, 2006). In contrast, similar issues contributing to phenotypic heterogeneity have been less widely considered, although they can cause substantial clinical variability between samples (Table 1 ). These include:

(a) Geographical origin. In addition to the likelihood that genetic contribution to illness varies between populations, there will be differences resulting from varying environmental exposures, sociocultural factors, service provision, etc.

(b) Ascertainment method. The spectrum of clinical features (symptoms, severity, functioning, illness course, etc.) of individuals recruited depends upon the mode of ascertainment. For example, inpatients at a tertiary referral centre differ from out-patients in secondary care.

(c) Unknown phenotypic model. Reliance on DSM-IV or ICD-10 categories obscures enormous clinical variability within categories. Perhaps of even greater concern, similarities across different categories of disorder are hidden.

(d) Measurement issues. Standardised methodologies for lifetime assessment of psychopathology are available. The same attention that is routinely given to technical issues of laboratory measurement must be given to correct, reliable and consistent use of phenotype measurement.

To maximise the potential of molecular genetic studies we need to pay much more attention to these phenotypic methodological issues than has recently been the norm.

\section{CONCLUSION}

There is a need to use a range of genetic approaches that are appropriate to identifying a spectrum of risk variants from the common through to the rare. McClellan et al (2007) argue for approaches targeting risk alleles of large individual effect but low population effect size. Although data from genetic epidemiology and molecular genetics support the existence of some rare chromosomal abnormalities of large effect size, the evidence suggests rare variants of large effect size do not account for the majority of cases of schizophrenia. However, one unanswered possibility is that most genetic risk results from rare alleles of moderate effect size. Since for the next few years common alleles of modest effect size are likely to be more tractable than are rare 
alleles, it seems appropriate that the focus in the immediate future will be on large samples and molecular genetic methods powered to detect the common alleles of modest effect size. Such approaches have only become available in the past $1-2$ years; it is far too early to judge whether they have been successful or not; indeed, at the time of writing, no whole genome-based surveys for common alleles of moderate effect size have been published. However, we predict that the interpretation of the data from such studies will be impeded by clinical variability across samples.

Replication of novel findings is essential. However, if as we suspect genetic variants that influence risk for psychiatric disorder influence aspects of the phenotype across DSM-IV/ICD-10 categories, and also influence only some aspects of the phenotype within these diagnostic categories, replication in psychiatric genetics will require close attention to both clinical psychiatric methodology and genetic methodology. We will need to become more sophisticated in our phenotypic thinking and move beyond studies and analyses based mainly on the traditional descriptive diagnostic categories (see Craddock \& Owen, 2005; Marneros, 2006; Angst, 2007). We need to ensure that clinical psychiatry is placed very firmly at the heart of psychiatric genetics.

\section{REFERENCES}

Angst, J. (2007) The bipolar spectrum. British Journal of Psychiatry, 190, 189-191.

Ben-Shachar, D. \& Laifenfeld, D. (2004)

Mitochondria, synaptic plasticity, and schizophrenia. International Review of Neurobiolology, 59, 273-296.

Blackwood, D. H., Fordyce, A., Walker, M.T., et al (200I) Schizophrenia and affective disorderscosegregation with a translocation at chromosome lq42 that directly disrupts brain-expressed genes: clinical and P300 findings in a family. American Journal of Human Genetics, 69, 428-433.

Botstein, D. \& Risch, N. (2003) Discovering genotypes underlying human phenotypes: past successes for mendelian disease, future approaches for complex disease. Nature Genetics, 33 (suppl.), 228-237.

Braff, D. L., Freedman, R., Schork, N. J., et al (2007) Deconstructing schizophrenia: an overview of the use of endophenotypes in order to understand a complex disorder. Schizophrenia Bulletin, 33, 21-32.

Cardon, L. R. \& Palmer, L. J. (2003) Population stratification and spurious allelic association. Lancet, 361, 598-604.

Chumakov, I., Blumenfeld, M., Guerassimenko, $\mathbf{O}$., et al (2002) Genetic and physiological data implicating the new human gene G72 and the gene for D-amino acid oxidase in schizophrenia. Proceedings of the National Academy of Science USA, 99, 13675-13680.

NICK CRADDOCK, PhD, FRCPsych, MICHAEL C. O'DONOVAN, PhD, FRCPsych, MICHAEL J. OWEN, PhD, FRCPsych, Department of Psychological Medicine, Wales School of Medicine, Cardiff University, Heath Park, Cardiff CFI4 4XN, UK

Correspondence: Professor Nick Craddock, Department of Psychological Medicine, Henry Wellcome Building, Wales School of Medicine, Cardiff University, Heath Park, Cardiff CFI4 4XN UK.

Email: craddockn@cardiff.ac.uk

(First received 23 November 2006, final revision 17 January 2007, accepted 18 January 2007)

Collins, F. S. (1992) Positional cloning: let's not call it reverse anymore. Nature Genetics, I, 3-6.

Craddock, N. \& Owen, M. J. (2005) The beginning of the end for the Kraepelinian dichotomy. British Journal of Psychiatry, 186, 364-366.

Craddock, N., Khodel, V., Van Eerdewegh, P., et al (1995) Mathematical limits of multilocus models: the genetic transmission of bipolar disorder. American Journal of Human Genetics, 57, 690-702.

Craddock, N., O'Donovan, M. C. \& Owen, M. J. (2005) The genetics of schizophrenia and bipolar disorder: dissecting psychosis. Journal of Medical Genetics, 42, 193-204.

Craddock, N., Owen, M. J. \& O'Donovan, M. C. (2006) The catechol-O-methyl transferase (COMT) gene as a candidate for psychiatric phenotypes: evidence and lessons. Molecular Psychiatry, II, 446-458.

Detera-Wadleigh, S. D. \& McMahon, F. J. (2006) G72/G30 in schizophrenia and bipolar disorder: review and meta-analysis. Biological Psychiatry, 60, 106-114.

Falconer, D. S. \& McKay, T. F. C. (1995) Introduction to Quantitative Genetics (4th edn). Longman.

Hamshere, M. L., Bennett, P., Williams, N., et a (2005) Genomewide linkage scan in schizoaffective disorder: significant evidence for linkage at Iq42 close to $\mathrm{DISCl}$, and suggestive evidence at 22qll and $19 \mathrm{pl} 3$. Archives of General Psychiatry, 62, 1081-1088.

Hattori, E., Liu, C., Badner, J. A., et al (2003) Polymorphisms at the G72/G30 gene locus, on 13q33, are associated with bipolar disorder in two independent pedigree series. American Journal of Human Genetics, 72 $|13|-\mid 140$.

Jablensky, A. (2006) Subtyping schizophrenia: implications for genetic research. Molecular Psychiatry, II, 815-836.

Lander, E. S. \& Schork, N. J. (1994) Genetic dissection of complex traits. Science, 30, 2037-2048.

McCarthy, M. I., Kruglyak, L. \& Lander, E. S. (1998)

Sib-pair collection strategies for complex diseases.

Genetic Epidemiology, I5, 317-340.

McClellan, J., Susser, E. \& King, M.-C. (2007) Schizophrenia: a common disease caused by multiple rare alleles. British Journal of Psychiatry, 190, 194-199.

McGue, M. \& Gottesman, I. I. (1989) A single dominant gene still cannot account for the transmission of schizophrenia, Archives of General Psychiatry, 46, 478-480

Malaspina, D. (200I) Paternal factors and schizophrenia risk: de novo mutations and imprinting. Schizophrenia Bulletin, 27, 379-393.

Margolis, R. L., Mclnnis, M. G., Rosenblatt, A., et al (1999) Trinucleotide repeat expansion and neuropsychiatric disease. Archives of General Psychiatry 56, $1019-103 \mid$

Marneros, A. (2006) Beyond the Kraepelinian dichotomy: acute and transient psychotic disorders and the necessity for clinical differentiation. British Journal of Psychiatry, 189, I-2.
Millar, J. K., Wilson-Annan, J. C., Anderson, S., et al (2000) Disruption of two novel genes by a translocation co-segregating with schizophrenia. Human Molecular Genetics, 9 , 1415-1423.

Moskvina, V., Craddock, N., Holmans, P., et al (2006) Effects of differential genotyping error rate on the type I error probability of case-control studies. Human Heredity, 6I, 55-64.

Muir, W. J., Pickard, B. S. \& Blackwood, D. H. (2006) Chromosomal abnormalities and psychosis. British Journal of Psychiatry, 188, 50I-503.

Munafo, M. R., Thiselton, D. L., Clark, T. G., et a (2006) Association of the NRGI gene and schizophrenia: a meta-analysis. Molecular Psychiatry, II, 539-546.

Owen, M. J., Craddock, N. \& O'Donovan, M. C. (2005) Schizophrenia: genes at last? Trends in Genetics, 2I, 518-525.

Plomin, R., Owen, M. J. \& McGuffin, P. (1994) The genetic basis of complex human behaviors. Science, $\mathbf{2 6 4}$ 1733-1739.

Risch, N. (1990) Linkage strategies for genetically complex traits. I. Multilocus models. American Journal of Human Genetics, 46, 222-228.

Risch, N. J. (2000) Searching for genetic determinants in the new millennium. Nature, 405, 847-856.

St Clair, D., Blackwood, D., Muir, W., et al (1990) Association within a family of a balanced autosomal translocation with major mental illness. Lancet, 336, 13-16.

Singh, S. M., Murphy, B. \& O'Reilly, R. (2002)

Epigenetic contributors to the discordance of monozygotic twins. Clinical Genetics, 62, 97-103.

Stefansson, H., Sigurdsson, E., Steinthorsdottir, V. et al (2002) Neuregulin I and susceptibility to schizophrenia. American Journal of Human Genetics, 7I 877-992.

Straub, R. E., Jiang, Y., MacLean, C. J., et al (2002) Genetic variation in the 6p22.3 gene DTNBPI, the human ortholog of the mouse dysbindin gene, is associated with schizophrenia. American journal of Human Genetics, 7I, 337-348.

Todd, J. A. (2006) Statistical false positive or true disease pathway? Nature Genetics, 38, 731-733.

Tosato, S., Dazzan, P. \& Collier, D. (2005) Association between the neuregulin I gene and schizophrenia: systematic review. Schizophrenia Bulletin, 31, 613-617.

Wang, W. Y., Barratt, B. J., Clayton, D. G., et al (2005) Genome-wide association studies: theoretical and practical concerns. Nature Reviews Genetics, 6, 109-118.

Williams, N. M., O'Donovan, M. C. \& Owen, M. J. (2005) Is the dysbindin gene (DTNBPI) a susceptibility gene for schizophrenia? Schizophrenia Bulletin, 31, 800805.

Williams, N. M., Green, E. K., Macgregor, S., et al (2006) Variation at the DAOA/G30 locus influences susceptibility to major mood episodes but not psychosis in schizophrenia and bipolar disorder. Archives of General Psychiatry, 63, 366-373. 